\title{
Southern Hemisphere VLBI Observations of the Expansion of GRO J1655-40
}

\author{
E.A. King, D.L. Jauncey, J.E. Reynolds, A.K. Tzioumis, D.J. McKay, \\ M.J. Kesteven, R. Gough, V. Migenes, M.W. Sinclair \\ Australia Telescope National Facility, PO Box 76, Epping, 2121, \\ Australia

\section{S.J. Tingay}

Mount Stromlo and Siding Spring Observatories, Canberra, ACT, 2611, Australia

R.A. Preston, D.L. Meier, D.W. Murphy, D.L. Jones

Jet Propulsion Laboratory, Pasadena, California 91109, USA

J.E.J. Lovel1, S.P. Ellingsen, P.M. McCulloch

Physics Department, University of Tasmania, Hobart, Tasmania, 7001, Australia

D. Campbell-Wilson, R.W. Hunstead

University of Sydney, NSW, 2006, Australia

J. Quick, D. Smits

Hartebeesthoek Radio Astronomy Observatory, Hartebeesthoek, 1740, South Africa

\begin{abstract}
We present high-resolution radio observations of the second Galactic superluminal radio source GRO1655-40, which was detected as an X-ray transient on 1994 July 27 . Our radio radio images reveal two components moving away from each other at an angular speed of $65 \pm 5$ mas/day, corresponding to superluminal motion $(v / c=1.4 \pm 0.4)$ at the estimated distance of 3-5 kpc. The 12-day delay between the X-ray and radio outbursts suggests that the ejection of material at relativistic speeds occurs during a stable phase of accretion onto a black hole, which follows an unstable phase with a high accretion rate. A complete description and discussion of these observations can be found in Tingay et al 1995 (Nature, 374, pp 141-143).
\end{abstract}




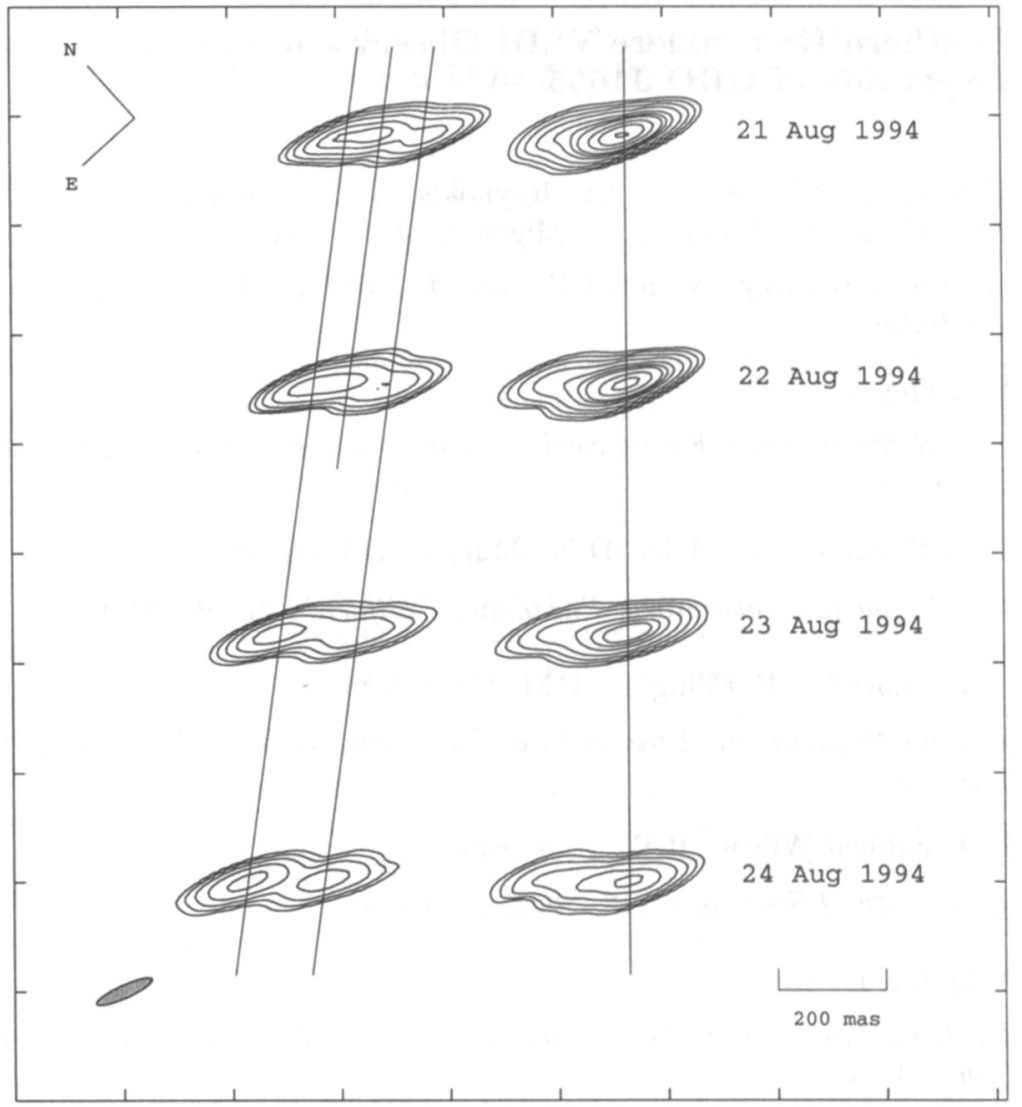

Figure 1. The four VLBI images at $2.29 \mathrm{GHz}$. The contour levels are $1,2,4,8,16,32,64,90,95 \%$ of the peak flux density in the montage of $0.59 \mathrm{Jy} \mathrm{beam}^{-1}$. The dynamic range is $100: 1$. The images have $1024 \times 1024$ pixels, each 2 mas across, and have each been rotated by $42^{\circ}$ and aligned to register the southwest component vertically. The restoring beam is $123 \times 26$ mas with major axis position angle of $72^{\circ}$. The observations included Australian antennas at Tidbinbilla, Parkes, Hobart, Culgoora and Mopra. Antennas were also used in the USA at Goldstone and Mauna Kea, and in South Africa at Hartebeesthoek, but the source was not detected on any intercontinental baseline. 